\title{
BMJ Open Effect of systemic bisphosphonate administration on patients with periodontal disease: a systematic review and meta-analysis protocol
}

Yoko Koide (D) , ${ }^{1}$ Yu Kataoka, ${ }^{2}$ Takeshi Hasegawa, ${ }^{3,4,5,6}$ Erika Ota, ${ }^{7,8}$ Hisashi Noma ${ }^{9}$

To cite: Koide Y, Kataoka Y, Hasegawa T, et al. Effect of systemic bisphosphonate administration on patients with periodontal disease: a systematic review and metaanalysis protocol. BMJ Open 2022;12:e057768. doi:10.1136/ bmjopen-2021-057768

- Prepublication history and additional supplemental material for this paper are available online. To view these files, please visit the journal online (http://dx.doi.org/10.1136/ bmjopen-2021-057768).

Received 26 September 2021 Accepted 08 February 2022

Check for updates

(C) Author(s) (or their employer(s)) 2022. Re-use permitted under CC BY-NC. No commercial re-use. See rights and permissions. Published by BMJ.

For numbered affiliations see end of article.

Correspondence to

Dr Yoko Koide;

koide64@dent.showa-u.ac.jp

\section{ABSTRACT}

Introduction Periodontal disease is a chronic oral infectious disease affecting adults worldwide as well as a lifestyle-related disease related to diabetes. Bisphosphonate is a drug often taken by patients with osteoporosis; however, it reportedly can cause jawbone necrosis. Due to its mechanism of action on bone tissue, bisphosphonate has been used topically on periodontal tissue to treat periodontal disease. However, the long-term systemic effects of bisphosphonates on periodontal tissues are unclear. This paper describes a protocol evaluating the effects of systemic bisphosphonate administration to prevent periodontal tissue destruction in patients with periodontal disease. No systematic review has attempted to summarise the evidence for systemic bisphosphonates in periodontal therapy. The results of the proposed systematic review will inform the practice and design of future clinical trials.

Methods and analysis This paper describes a protocol for a systematic review of the relevant published analytic research using an aggregative thematic approach according to the Preferred Reporting Items for Systematic Reviews and Meta-Analyses Protocols guidelines. Two authors will perform a comprehensive search for studies on Medline/PubMed, Scopus, Embase, LILACS and the Cochrane Central Register of Controlled Trials databases. Abstract screening, full-text screening and data extraction will be performed independently by two authors. A metaanalysis will be conducted as appropriate.

Ethics and dissemination The protocol of this systematic review will be provided in a peer-reviewed journal. Formal ethics approval is not necessary because researchers will not identify individuals in the report.

PROSPERO registration number CRD42020212698 (http://www.crd.york.ac.uk/PROSPER0/).

\section{INTRODUCTION}

\section{Description of the condition}

Periodontal disease is a chronic inflammatory disease caused by anaerobic periodontopathic bacteria, resulting in infection of the periodontal tissues and progressive resorption of the alveolar bone. Most children worldwide have symptoms of gingivitis, and many adults have an early stage of periodontal disease. In addition, $13.1 \%$ of adults aged $35-44$ years

\section{Strengths and limitations of this study}

- This systematic review protocol complies with the Preferred Reporting Items for Systematic Reviews and Meta-Analyses Protocols (PRISMA-P) Checklist.

- This study will be conducted in strict accordance with the recommendations of the Cochrane Handbook for a systematic review.

- In the preparation of this protocol, our study is registered with the international prospective register of systematic reviews (PROSPER0).

- It is not possible to provide a comprehensive analysis of all research outputs, including unpublished papers, such as Grey literature and conference abstracts.

have a community periodontal index of 4 , which indicates severe periodontitis that can lead to tooth loss. Aggressive periodontitis in young people affects $2 \%$ of the population and affects adolescents with early tooth loss. ${ }^{1}$

Osteoporosis is a skeletal disease characterised by decreased bone strength and an increased risk of fracture. The decrease in oestrogen due to menopause promotes bone resorption and is one of the causes of osteoporosis. Furthermore, it has been reported that there is a relationship between bone fragility and metabolic syndromes, such as diabetes, hypertension, dyslipidaemia and obesity. The bone fracture risk is reportedly increased even though bone density is maintained, especially in type 2 diabetes. ${ }^{2}$ Chronic obstructive pulmonary disease and chronic kidney disease can potentially increase the risk of fracture. In these ageing-associated systemic metabolic disorders, it is possible that weakening of the bone matrix due to abnormal collagen metabolism, as well as the decrease in bone mineral, is the aetiology of osteoporosis. Osteoporosis and associated fractures are a major cause of disease, disability and death in older people and a 
global problem requiring huge healthcare costs. The annual number of hip fractures worldwide is estimated to increase from 1.7 million in 1990 to approximately 6.3 million by 2050 . The lifetime risk of osteoporotic fractures in women is approximately $40 \%$. In contrast, the risk is only $13 \%$ in men. ${ }^{3}$ Globally, however, the incidence of proximal femur fractures has increased since the 2000 survey in European countries, the USA, Canada, Oceanian countries and Japan. ${ }^{4}$

Recent advances in drug therapy have made it possible to reduce the risk of osteoporotic fractures, and it is clear that bone resorption inhibitors such as bisphosphonates, antireceptor activators of the NF-kappa B ligand (RANKL) antibody, and selective oestrogen receptor modulators (SERMs) lower the increased risk of bone fractures. ${ }^{56}$ Because of its cost-effectiveness, bisphosphonate is widely used as a therapeutic drug for osteoporosis. ${ }^{7-10}$

\section{Description of the intervention}

Currently, therapeutic agents for osteoporosis include bone resorption inhibitors such as bisphosphonates, anti-RANKL antibodies, SERM, calcium drugs, female hormone drugs, active vitamin $\mathrm{D}$ preparations and vitamin K. Bisphosphonates are often used in postmenopausal women as therapeutic agents for osteoporosis. As an adjunct to conventional periodontal therapy, host modulatory therapy has been performed experimentally for several years. In host modulatory therapy, bisphosphonates are administered directly into the periodontal pocket between the tooth and the gingiva, or as part of periodontal tissue regeneration therapy, a method of administering bisphosphonate directly to bone defects in the jaw during surgery. ${ }^{11}{ }^{12}$ However, the effects of bisphosphonate as a host modulatory therapy in periodontal treatment have not been previously reported. The present study will focus on the alveolar bone effects in patients who received systemic bisphosphonates to treat various diseases such as osteoporosis.

\section{How these drugs might work}

Bisphosphonates are the first-choice drug in treating osteoporosis and are often used to treat hypercalcaemia, bone metastasis of malignant tumours, and Paget's disease of bone (osteitis deformans). Bisphosphonates have a high affinity for calcium phosphate and are known to inhibit osteoclast activity. Bisphosphonates are also thought to inhibit the activity of matrix metalloproteinases by a mechanism involving the chelation of cations. ${ }^{13}$ The adjunctive effect of bisphosphonates in the control of alveolar bone destruction has been demonstrated in both spontaneous and experimental models in vivo. ${ }^{14} 15$ However, many cases of bisphosphonate-related osteonecrosis of the jaw have been reported.

\section{Why is this review important?}

As noted above, there are many patients with osteoporosis who are taking bisphosphonates as well as patients with the periodontal disease worldwide. Previous systematic reviews have examined the effectiveness of bisphosphonates in periodontal treatment. ${ }^{16}{ }^{17}$ However, more recent systematic reviews reported that the older systematic reviews did not consider differences in the route of bisphosphonate administration, such as systemic administration, intraperiodontal pocket administration and administration into bone defects during periodontal surgery. Therefore, past systematic reviews could not clarify the effectiveness of long-term systemic administration of bisphosphonates for periodontal disease. A few systematic reviews of bisphosphonates for patients with periodontal disease have been published; however, no meta-analysis limited to the effect of systemic bisphosphonate administration in patients with periodontal disease has been published.

\section{OBJECTIVES}

This systematic review and meta-analysis will examine the effects of systemic bisphosphonate administration to prevent the breakdown of periodontal tissue in patients with periodontal disease.

\section{METHODS AND ANALYSIS \\ Methods}

This protocol is compliant with the standards of the Preferred Reporting Items for Systematic Review and Meta-Analysis Protocols (PRISMA-P) statement ${ }^{18}$ and is registered with the International Prospective Register of Systematic Reviews (PROSPERO) database (\#CRD42020212698; first version: 4 November 2020, second version: 6 September 2021). ${ }^{19}$ This study will be conducted starting in February 2022, with an intended completion date in September 2022.

\section{Criteria for considering studies for this review Types of studies}

We decided to include randomised, placebo-controlled trials and individual and cluster randomised controlled trials (RCTs) with a minimum follow-up of 6 months. We will exclude non-randomised studies, quasi-RCTs, in vitro studies, animal studies, extended abstracts and observational studies. The details of the study's inclusion and exclusion criteria are shown in online supplemental additional file 1.

\section{Types of participants}

We will include adults ( $\geq 18$ years of age) diagnosed with periodontal disease. Furthermore, the subjects will be periodontal patients who are currently taking bisphosphonates for the following illnesses: osteoporosis, multiple myeloma, Paget's disease, malignant hypercalcemia, osteogenesis imperfecta, suppression of progressive ectopic ossification after traumatic nerve injury, severe neuropathy and hip arthroplasty. There were no restrictions on the choice of patients, depending on their general condition. 


\section{Types of interventions}

We will include studies on the systemic use of administered bisphosphonates in combination with periodontal therapy. Some patients may not be taking bisphosphonates systemically for fear of adverse effects. We will include a control group, that is, patients receiving periodontal therapy combined with a placebo. The control group will also include those who received only periodontal therapy without taking placebo tablets, such as an open trial. Patients taking vitamin D instead of bisphosphonates will also be included in the control group. In this study, periodontal therapy will be defined as non-surgical initial therapy with some level of subgingival debridement and/ or root plaining and periodontal surgery. The same protocol will be applied to both test and control groups.

\section{Types of outcome measures}

We will create a table of summary findings on the following primary and secondary outcomes.

1. Primary outcome

Reduction in probing depth compared with pretreatment (baseline) and post-treatment follow-up. The time of measurement during follow-up will be recorded.

2. Secondary outcomes

We will also examine the following outcomes at the same measurement time as the primary outcomes:

- the gain in clinical attachment level,

- changes in bleeding on probing,

- changes in radiographic bone defect fill,

- numbers of missing teeth,

- changes in plaque indices,

- and patient-reported outcomes including adverse events.

\section{Search methods for identification of studies}

To identify studies for inclusion in this review, the following electronic databases will be searched for relevant articles published up to November 2021: Medline/ PubMed, Scopus, Embase, LILACS and the Cochrane Central Register of Controlled Trials. There will not be any language or publication period limitations on our searches. Non-English publications will be translated by accessing translation services available from Showa University or staff or doctoral/postdoctoral research students who are native speakers at Showa University or St. Luke's International University. A search strategy was designed using the advice of a librarian with experience in systematic reviews. Key search terms (MeSH and keyword terms) will include the following: periodontal disease, periodontitis, diphosphonates, bisphosphonate and RCTs. We will perform this search (online supplemental additional file 2) electronically.

\section{Data collection}

Selection of studies

We will use Endnote software (EndNoteX9; Thomson Reuters) to collect citations and remove any duplicate articles. Two authors (YKo and YKa) will independently screen titles and abstracts (when available) for all reports obtained by electronic and manual searches which have been stored in the software programme. Studies that do not meet the inclusion criteria will be discarded (online supplemental additional file 1), and the reasons for exclusion will be described in a table. After the initial phase, the authors (YKo and YKa) will independently review the full-text articles of the remaining studies for inclusion and exclusion in the final analysis. Any disagreements will be resolved by discussion and referral to a third author $(\mathrm{TH})$. The process of study selection will be demonstrated in the PRISMA flow chart.

\section{Data extraction and management}

The two authors will independently perform data extraction on the following outcomes:

- Study information

- Authors

- Country

- Publication year

- Journal titles

- Other (eg, pharmaceutical sponsorship data)

- Trial period

- Study settings (eg, hospital or clinic, multicentric study, or single-centre study)

- Study methods

- Study design

- Randomisation method

- Definition of periodontal disease

- Participants:

- Numbers of participants

- Numbers of participants who dropped out

- Eligibility and exclusion criteria

- Age and sex

- Diagnosis of periodontal disease at first examination

- Smoking status (current smoker, past smoker and never smoker)

- Information of periodontal treatment

- Systemic general condition

- Interventions

- Drug name used (dosage)

- Route of administration

- Administration period

- Control details

- Outcomes

- Primary outcome

- Secondary outcomes

- Analytical methods

- Results

The two authors (YKo and YKa) will resolve disagreements by discussion and consultation with a third author (TH), if necessary.

\section{Assessment of risk of bias in included studies}

Risk of bias evaluation of all included papers will be performed by two authors (YKo and YKa) individually using tools as described in Chapter 8 of the Cochrane Handbook for Systematic Reviews of Interventions Version 6.2 (updated February 2021).$^{20}$ The third author (TH) will resolve any 
disparities between the primary reviewers. Differences in the assessment of the risk of bias will be resolved by a fourth reviewer (EO). We will assess the following domains of the risk of bias: (1) sequence generation, (2) allocation concealment, (3) blinding of participants and personnel, (4) blinding of outcome assessment, (5) incomplete outcome data, (6) selective outcome reporting and (7) other biases such as baseline imbalance.

\section{Strategy for data synthesis}

We will perform a meta-analysis to assess the included studies' clinical and methodological diversity and statistical heterogeneity. We will also use a DerSimonian-Laird-type random-effects model to estimate the pooled effects by metaanalysis, and account for statistical heterogeneity between the included studies. For continuous outcomes, we will use the mean difference or standardised mean difference as appropriate. In addition, for dichotomous outcomes, we will adopt the risk difference or risk ratio as appropriate. Where quantitative integration is not possible, the results will be analysed and described. If we can statistically pool the results, we will provide forest plots to summarise the results of individual studies. For data synthesis, we use Review Manager (RevMan) software, V.5.4 (Cochrane Collaboration, Oxford, UK).

\section{Measurement of treatment effect}

The mean difference or standardised mean difference and 95\% CIs for the difference between the intervention and comparison groups for continuous outcomes will be calculated as appropriate. In addition, for dichotomous outcomes, the risk difference or risk ratio and $95 \% \mathrm{CI}$ will be calculated as appropriate.

\section{Dealing with missing data}

In the case of missing data, we will perform a sensitivity analysis to confirm the impact of including studies with substantial levels $(>20 \%)$ of missing data in all assessments of the intervention effect.

\section{Assessment of heterogeneity}

We will apply the DerSimonian-Laird-type random-effect model for synthesis analyses to address heterogeneity between studies. Statistical heterogeneity among the trials will be evaluated using the heterogeneity variance $\tau^{2}$, Higgins' ${ }^{2}$ statistic and the Q-statistic (Cochrane's test). Further, we will conduct graphical analyses for the forest plots that assess overlaps of the confidence intervals for individual studies to find possible heterogeneity. To assess clinical heterogeneity by potential effect modifiers, we will conduct subgroup analyses or metaregression analyses for five variables indicated in the Subgroup analysis section. We will consider heterogeneity significant if $\mathrm{p}<0.1$. We will also consider the following categories of $\mathrm{I}^{2}$ statistics: $<25 \%, 25 \%-50 \%$ and $50 \%-75 \%$. In the case of $\mathrm{I}^{2}>50$, we will examine the heterogeneity using subgroup regression or metaregression. If we identify the reasons for heterogeneity, we will report accordingly. If heterogeneity is not resolved, we will pool the results for $\mathrm{I}^{2}$ between $50 \%$ and $75 \%$, and include the note on the heterogeneity. If $\mathrm{I}^{2}>75 \%$, the results will not be pooled. We will pool the outcome data using a random-effect model. We will also use the Distiller SR and RevMan for data extraction and meta-analysis. We will show the forest plots to assess statistical heterogeneity, describing the direction and magnitude of the effect and the overlap of CIs.

\section{Assessment of reporting biases}

We will present the results of a funnel plot to assess publication bias when a meta-analysis is performed in a sufficient number of studies (more than 10).

\section{Subgroup analysis}

If we identify substantial heterogeneity, subsets or subgroups will be analysed as follows:

- different pathology-administered bisphosphonates (osteoporosis, multiple myeloma, Paget's disease, malignant hypercalcemia, osteogenesis imperfecta, suppression of progressive ectopic ossification after traumatic nerve injury, severe neuropathy and hip arthroplasty),

- duration of the intervention (3 months ${ }^{21}$ vs 6 months, ${ }^{21-23} 1$ year, ${ }^{24} 2$ years, ${ }^{25} 26$ and 3 years ${ }^{27}$ ),

- different drug/dosages (alendronate vs neridronic acid, zoledronic acid, and risedronate; alendronate at $10 \mathrm{mg} /$ day $^{2324}$ vs $70 \mathrm{mg}$ once per week, ${ }^{22} 25$ neridronic acid at $12.5 \mathrm{mg}$ once per week, ${ }^{21}$ zoledronic acid at $5 \mathrm{mg}$ once per year ${ }^{26} 27$ vs $5 \mathrm{mg} /$ day and risedronate at $5 \mathrm{mg} /$ day $^{24}$ ),

- difference in periodontal therapy (no treatment (only oral hygiene instruction) ${ }^{25-27}$ vs non-surgical initial therapy, ${ }^{21-24}$ periodontal surgery),

- study design (double blind ${ }^{23242627}$ vs open label $^{21}$ ).

Sensitivity analysis

We will perform a sensitivity analysis to check the risk for primary outcomes, excluding studies with a high risk of bias (allocation concealment and incomplete outcome data).

\section{Summary of findings and assessment of the certainty of the evidence}

We will create a table of summary findings on primary and secondary outcomes according to the procedure described in the Cochrane Handbook for Systematic Reviews of Interventions. We will assess the quality of evidence involved in the primary outcomes using the Grading of Recommendations Assessment, Development and Evaluation approach.

\section{Patient and public involvement}

The protocol of this systematic review will not involve patients and the public. This study has no plans to involve patients or the public in the design, implementation, reporting or dissemination planning.

\section{ETHICS AND DISSEMINATION}

The protocol of this systematic review will be published in a peer-reviewed journal and presented at relevant national and international conferences. 
Formal ethics approval is not necessary because researchers will not identify individuals in the report. This protocol for this study has registered with PROSPERO (CRD42020212698, http://www.crd.york. ac.uk/PROSPERO/). ${ }^{19}$ The effects of systemically administered bisphosphonates on the progression of periodontal disease analysed in this review will serve as evidence to aid in the selection of this drug by patients who may be concerned about its side effects. The final analysis will be published and subsequently disseminated on university and social media platforms. The results will also be presented at conferences, and we will submit the research findings in peer-reviewed journals.

\section{Author affiliations}

${ }^{1}$ Department of Periodontology, Showa University School of Dentistry, Ota-ku, Tokyo, Japan

${ }^{2}$ Division of Biomaterials and Engineering, Department of Conservative Dentistry, Showa University School of Dentistry, Shinagawa-ku, Tokyo, Japan

${ }^{3}$ Showa University Research Administration Center (SURAC), Showa University, Shinagawa-ku, Tokyo, Japan

${ }^{4}$ Division of Nephrology, Department of Medicine, Showa University School of Medicine, Shinagawa-ku, Tokyo, Japan

${ }^{5}$ Department of Hygiene, Public Health and Preventive Medicine, Showa University School of Medicine, Shinagawa-ku, Tokyo, Japan

${ }^{6}$ Center for Innovative Research for Communities and Clinical Excellence,

Fukushima Medical University, Fukushima, Fukushima, Japan

${ }^{7}$ Graduate School of Nursing Science, Global Health Nursing, St Luke's International University, Chuo-ku, Tokyo, Japan

${ }^{8}$ The Tokyo Foundation for Policy Research, Minato-ku, Tokyo, Japan

${ }^{9}$ Department of Data Science, Institute of Statistical Mathematics, Tachikawa,

Tokyo, Japan

Contributors Conception: YKo. Design of the work: YKo, YKa, TH, EO. Statistical analysis: HN. Interpretation of data: all authors. Drafting or revising the manuscript: YKo, TH, EO, HN.

Funding This systematic review was undertaken as part of a project organised by the Showa University Research Administration Centre. This work was supported by JSPS KAKENHI (Grant Number 19K03092). JSPS KAKENHI is a competitive fund funded by the Japanese government. Therefore, JSPS KAKENHI does not play a role in the development protocol.

Competing interests None declared.

Patient consent for publication Not applicable.

Provenance and peer review Not commissioned; externally peer reviewed.

Supplemental material This content has been supplied by the author(s). It has not been vetted by BMJ Publishing Group Limited (BMJ) and may not have been peer-reviewed. Any opinions or recommendations discussed are solely those of the author(s) and are not endorsed by BMJ. BMJ disclaims all liability and responsibility arising from any reliance placed on the content. Where the content includes any translated material, BMJ does not warrant the accuracy and reliability of the translations (including but not limited to local regulations, clinical guidelines, terminology, drug names and drug dosages), and is not responsible for any error and/or omissions arising from translation and adaptation or otherwise.

Open access This is an open access article distributed in accordance with the Creative Commons Attribution Non Commercial (CC BY-NC 4.0) license, which permits others to distribute, remix, adapt, build upon this work non-commercially, and license their derivative works on different terms, provided the original work is properly cited, appropriate credit is given, any changes made indicated, and the use is non-commercial. See: http://creativecommons.org/licenses/by-nc/4.0/.

\section{ORCID iD}

Yoko Koide http://orcid.org/0000-0002-6395-4704
REFERENCES

1 Malmö University. Periodontal disease. Available: https://capp.mau. se/periodontal-disease/ [Accessed 10 Sep 2021].

2 Yamamoto M, Yamaguchi T, Yamauchi M, et al. Diabetic patients have an increased risk of vertebral fractures independent of BMD or diabetic complications. J Bone Miner Res 2009;24:702-9.

3 World Health Organization. Nutrition for older persons. Available: https://www.who.int/nutrition/topics/ageing/en/ [Accessed $10 \mathrm{Sep}$ 2021].

4 Harvey N, Dennison E, Cooper C. Osteoporosis: impact on health and economics. Nat Rev Rheumatol 2010;6:99-105.

5 Lin T, Wang C, Cai X-Z, et al. Comparison of clinical efficacy and safety between denosumab and alendronate in postmenopausal women with osteoporosis: a meta-analysis. Int J Clin Pract 2012;66:399-408.

6 Migliore A, Broccoli S, Massafra U, et al. Ranking antireabsorptive agents to prevent vertebral fractures in postmenopausal osteoporosis by mixed treatment comparison meta-analysis. Eur Rev Med Pharmacol Sci 2013;17:658-67.

7 Goeree R, Blackhouse G, Adachi J. Cost-effectiveness of alternative treatments for women with osteoporosis in Canada. Curr Med Res Opin 2006;22:1425-36.

8 Pfister AK, Welch CA, Lester MD, et al. Cost-effectiveness strategies to treat osteoporosis in elderly women. South Med J 2006;99:123-31.

9 Stevenson M, Jones ML, De Nigris E, et al. A systematic review and economic evaluation of alendronate, etidronate, risedronate, raloxifene and teriparatide for the prevention and treatment of postmenopausal osteoporosis. Health Technol Assess 2005;9:1-160.

10 Tosteson ANA, Burge RT, Marshall DA, et al. Therapies for treatment of osteoporosis in US women: cost-effectiveness and budget impact considerations. Am J Manag Care 2008;14:605-15.

11 Donos N, Calciolari E, Brusselaers N, et al. The adjunctive use of host modulators in non-surgical periodontal therapy. A systematic review of randomized, placebo-controlled clinical studies. J Clin Periodontol 2020;47 Suppl 22:199-238.

12 Golub LM, Lee H-M. Periodontal therapeutics: current hostmodulation agents and future directions. Periodontol 2000 2020;82:186-204.

13 Özdemir SP, Kurtiș B, Tüter G, et al. Effects of low-dose doxycycline and bisphosphonate clodronate on alveolar bone loss and gingival levels of matrix metalloproteinase- 9 and interleukin- $1 \beta$ in rats with diabetes: a histomorphometric and immunohistochemical study. $J$ Periodontol 2012;83:1172-82.

14 Alencar VBM, Bezerra MM, Lima V, et al. Disodium chlodronate prevents bone resorption in experimental periodontitis in rats. $J$ Periodontol 2002;73:251-6.

15 Weinreb M, Quartuccio H, Seedor JG, et al. Histomorphometrical analysis of the effects of the bisphosphonate alendronate on bone loss caused by experimental periodontitis in monkeys. J Periodontal Res 1994;29:35-40.

16 Akram Z, Abduljabbar T, Kellesarian SV, et al. Efficacy of bisphosphonate as an adjunct to nonsurgical periodontal therapy in the management of periodontal disease: a systematic review. $\mathrm{Br} \mathrm{J}$ Clin Pharmacol 2017;83:444-54.

17 Reddy MS, Geurs NC, Gunsolley JC. Periodontal host modulation with antiproteinase, anti-inflammatory, and bone-sparing agents. A systematic review. Ann Periodontol 2003;8:12-37.

18 Shamseer L, Moher D, Clarke M, et al. Preferred reporting items for systematic review and meta-analysis protocols (PRISMA-P) 2015: elaboration and explanation. BMJ 2015;350:g7647.

19 Koide Y, Kataoke Y, Hasegawa T. The effect of systemic bisphosphonate administration on periodontal disease patients: a systematic review. Available: https://www.crd.york.ac.uk/prospero/ display_record.php?ID=CRD42020212698 [Accessed 10 Sep 2021].

20 Cochrane Training. Cochrane Handbook for systematic reviews of interventions version 6.2. Available: http://www.training.cochrane. org/handbook [Accessed 10 Sep 2021].

21 Graziani F, Cei S, Guerrero A, et al. Lack of short-term adjunctive effect of systemic neridronate in non-surgical periodontal therapy of advanced generalized chronic periodontitis: an open labelrandomized clinical trial. J Clin Periodontol 2009;36:419-27.

22 El Kilani NS, Hazzaa HH, Hemoud HES, et al. Clinical and radiographic assessment of single or combined treatment with Lepidium sativum and alendronate of Non-Surgically treated chronic periodontitis in postmenopausal osteoporotic women. J Int Acad Periodontol 2019;21:20-8.

23 Rocha ML, Malacara JM, Sánchez-Marin FJ, et al. Effect of alendronate on periodontal disease in postmenopausal women: a randomized placebo-controlled trial. J Periodontol 2004;75:1579-85. 
24 Lane N, Armitage GC, Loomer P, et al. Bisphosphonate therapy improves the outcome of conventional periodontal treatment: results of a 12-month, randomized, placebo-controlled study. J Periodontol 2005;76:1113-22.

25 Jeffcoat MK, Cizza G, Shih WJ, et al. Efficacy of bisphosphonates for the control of alveolar bone loss in periodontitis. J Int Acad Periodontol 2007;9:70-6.
26 Taguchi A, Shiraki M, Tanaka S, et al. Improved periodontal disease and prevention of tooth loss in osteoporosis patients receiving once-yearly zoledronic acid: a randomized clinical trial. Menopause 2019;26:1277-83.

27 Grbic JT, Landesberg R, Lin S-Q, et al. Incidence of osteonecrosis of the jaw in women with postmenopausal osteoporosis in the health outcomes and reduced incidence with zoledronic acid once yearly pivotal fracture trial. J Am Dent Assoc 2008;139:32-40. 